Paper

\title{
Dynamic behaviour of a ring coupled boost converter system with passivity-based control
}

\author{
Rutvika Manohar ${ }^{1 a)}$ and Takashi Hikihara ${ }^{1 b)}$ \\ ${ }^{1}$ Department of Electrical Engineering, Kyoto University \\ Katsura, Nishikyoku, Kyoto 615-8510, Japan \\ a)r-manohar@dove.kuee.kyoto-u.ac.jp \\ b) hikihara.takashi.2n@kyoto-u.ac.jp
}

Received June 2, 2019; Revised October 7, 2019; Published January 1, 2020

\begin{abstract}
This paper proposes a dispersed generation system of multiple DC/DC converters with DC power sources connected in a ring formulation. Here is presented the analysis of the system based on the stored energy and passivity characteristics. Passivity Based Control $(\mathrm{PBC})$, with its energy-modifying and damping-injection technique, is applied to a ring coupled converter system to stabilize itself at a desired DC voltage in the presence of external disturbances. The numerical results reveal the effective application of the control as a robust and flexible technique.
\end{abstract}

Key Words: dispersed generation, ring coupled converters, boost converter, passivity-based control

\section{Introduction}

Generation and storage of electricity with non-conventional power sources like photovoltaic cells, fuel cells, wind power, and battries are realized with distributed generation [1]. Power converters are seen as an important interface to transfer electricity from renewable sources of energy into the power network. Towards designing a dispersed generation system,which is autonomous from the conventional power grid, we propose a DC/DC converter system with DC power sources connected in a ring formulation and coupled with dissipation. Ring formulation is proposed to represent one of the ways to realize distributed autonomous systems in small residential areas [2]. The basic idea of such an 'autonomous distributed generation system' is illustrated in Fig. 1. This configuration is useful in harnessing energy from DC sources of electricity like solar cells, batteries, fuel cells, and so on.

Passivity Based Control (PBC) aims at stabilizing the system by modifying the potential and kinetic energy functions. It stems from the fundamental notion of 'passivity', a property physically corresponding to the energy of the system. Passive systems hold the characteristic that the energy stored is always less than the energy supplied from outside [3]. PBC applied to passive systems is performed in two stages. The first stage consists of shaping of the potential energy of the system such that the shaped potential energy function has a global and unique minimum at the desired 


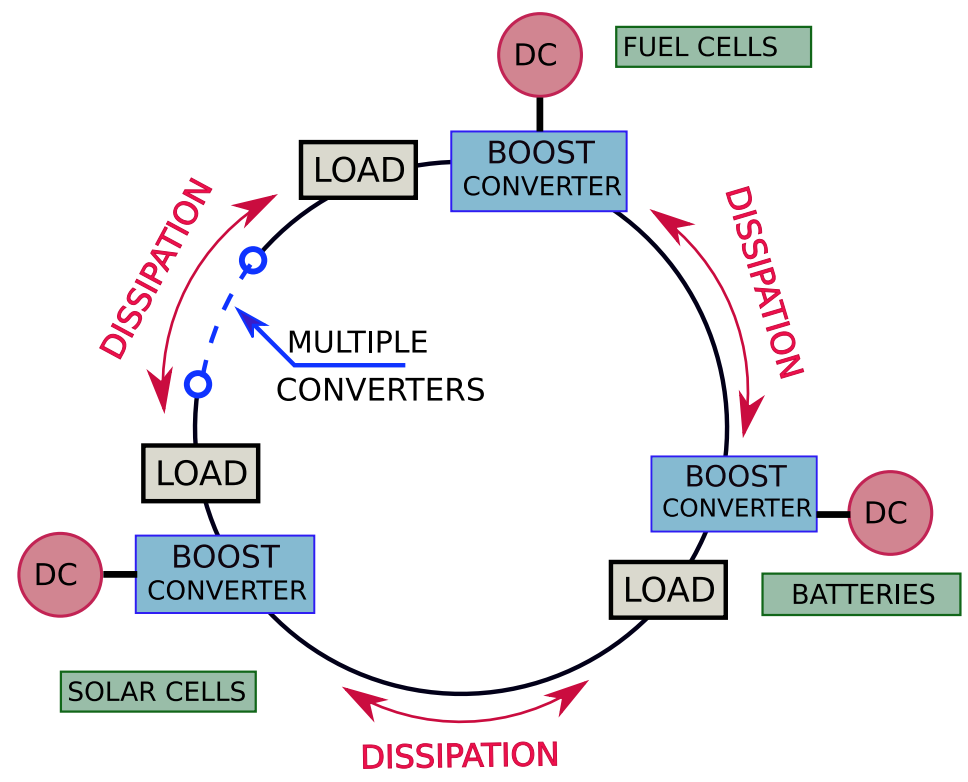

Fig. 1. System configuration.

equilibrium. The second stage is to add damping to make the system exponentially stable [4].

The concept of passive systems was first developed in [5] in the context of electrical circuits. This, along with the framework of dissipative systems developed in [6] helps to understand the concepts of passive systems and passivity based control from a dynamical systems point of view.

The energy-shaping plus damping-injection methodology, used to solve state feedback set point regulation problems in fully actuated robotic systems, was developed by Takegaki and Arimoto. The method has evolved into 'Passivity Based Control' [7]. The term 'Passivity Based Control' was introduced first in [8] to define a control methodology whose objective was to render the closed loop passive. This control objective was first employed for the adaptive control of robot manipulators by [9].

Passivity Based Control has been traditionally applied to Euler-Lagrange systems [4]. Here, the energy shaping stage accomplishes shaping of the potential energy and keeping the original kinetic energy to satisfy the passivation objective. For electronic systems which consist of non-energy elements like dissipation through resistance and switching elements, Lagrangian formulation is difficult or, at times, impossible to implement [4]. For these systems, the kinetic energy as well as potential energy of the system need to be modified to design a storage function. Considering these restrictions, instead of Euler-Lagrange modelling, Port-Controlled Hamiltonian modelling (PCHM) is appropriate to achieve better results with PBC [10]. The modified storage function can be a candidate of Lyapunov function.

The application of PBC to DC/DC converters was given in [11]. Then, the application of PBC for parallely connected DC/DC converters was discussed in [12]. This paper discusses the method to use $\mathrm{PBC}$ to a distributed system, a ring coupled DC/DC converter system with multiple DC inputs. The DC/DC converter discussed in this paper is a boost converter, the characteristics of which have been well documented [13].

For a ring-coupled converter system introduced in this paper, it is confirmed that the converters keep the property of passivity, individually. In addition, the feedback control is employed with minimizing the energy function of the entire system, as opposed to the individual energy function of a single DC/DC converter. The energy transfer between the individual converter units governs the dynamic behaviour of the whole ring coupled system. The application of PBC, with 'energy shaping' of the entire system, manifests the property of robustness despite the flow of energy between individual elements. 


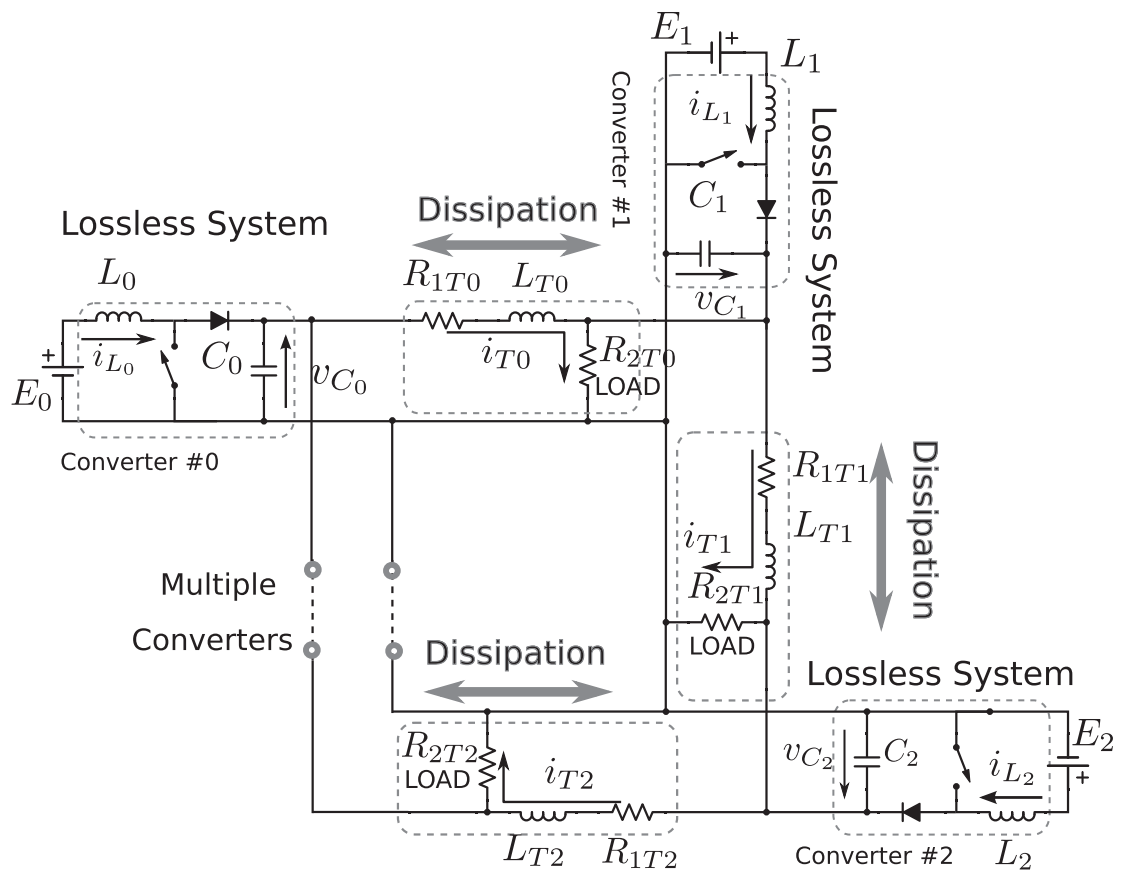

Fig. 2. Schematic diagram of ring coupled converters.

\section{System design and modelling}

\subsection{System equations}

We introduce a system consisting of multiple boost converters with multiple DC input sources. These converters are coupled together with dissipation through inductance and resistance. The coupling through dissipation represents a transmission line model, with inductive and resistive elements. The basic configuration is shown in Fig. 1.

The schematic diagram of the circuit is shown in Fig. 2. The coupled converters are responsible for the constant voltage output in the ring. Here, load resistances $\left(R_{2 T \mathrm{n}}\right)$ are across the output voltages, where as the dissipation elements of line inductor $\left(L_{T \mathrm{n}}\right)$ and line resistance $\left(R_{1 T \mathrm{n}}\right)$ are in series. The capacitor in the transmission line model is considered as negligible. This is owing to the fact that a parallel capacitor is dominant in the boost converter configuration. The number of converters was set such that it enhances the asymmetry of the system. The number of converters in the ring does not cause any loss of generality. Then, the objective is to apply PBC to the whole system, including the dissipation between the converters.

The system equations are given by Eqs. (1)-(3). Subscription n denotes the index of the converter. $u_{\mathrm{n}}$ denotes the switch position for the $\# \mathrm{n}$ converter. $u_{\mathrm{n}}=1$ implies that the switch is $\mathrm{ON}$ and $u_{\mathrm{n}}=0$, that it is OFF. $L_{T \mathrm{n}}$ is the inductance and $R_{1 T \mathrm{n}}$ is the dissipation between the \#n and $\# \mathrm{n}+1$ converter.

$$
\begin{aligned}
L_{\mathrm{n}} \dot{i}_{L \mathrm{n}} & =-\left(1-u_{\mathrm{n}}\right) v_{C \mathrm{n}}+E_{\mathrm{n}} \\
C_{\mathrm{n}} \dot{v}_{C \mathrm{n}} & =\left(1-u_{\mathrm{n}}\right) i_{L \mathrm{n}}-i_{T \mathrm{n}}+i_{T(\mathrm{n}-1)}-\frac{v_{C \mathrm{n}}}{R_{2 T(\mathrm{n}-1)}} \\
L_{T \mathrm{n}} \dot{i}_{T \mathrm{n}} & =v_{C \mathrm{n}}-v_{C(\mathrm{n}+1)}-R_{1 T \mathrm{n}} i_{T \mathrm{n}}
\end{aligned}
$$

Here, the dot notation represents differentiation with respect to time.

\subsection{Port Controlled Hamiltonian Modelling}

Port Controlled Hamiltonian Modeling(PCHM) is the network representation of systems in interaction with their environment [14]. The Hamiltonian approach allows for the systematic modelling of electrical systems including resistors and switching elements. The non-energetic terms are extracted from the circuit. It leaves the energy conserving LC circuit. The non-energy elements are then introduced into the circuit in the form of 'ports' [14]. Such configuration of LC circuits with ports is 
represented as a generalized Hamiltonian system with external input variables. PCHM technique is used for modeling DC/DC converters, to allow for the inclusion of power electronic switches and load resistances.

PCHM classifies the system neatly into physically well defined interconnection $(\mathbf{J})$, dissipation $(\mathbf{R})$, and external input (E) matrices within a state space framework [4]. The system model using the Port Controlled Hamiltonian(PCH) framework is given by Eq. (4) as in [15].

$$
\mathbf{D} \dot{\boldsymbol{x}}(t)=[\mathbf{J}-\mathbf{R}] \frac{\partial H}{\partial x}+\mathbf{E}
$$

For $\mathrm{m}$ converters in the configuration, $\boldsymbol{x}$, the state of the system, is a column matrix $((\mathrm{m} \times 3) \times 1)$ of all the inductor currents and capacitance voltages. The matrix $\mathbf{D}$ is a diagonal matrix of the capacitances and inductances of the corresponding currents and voltages. $\mathbf{J}$ gives the interconnection, and $\mathbf{R}$ the dissipation in the system. The interconnection is a function of $u_{\mathrm{n}}$, the switch position of the corresponding boost converters in the ring configuration. $\mathbf{E}$, the input matrix is a column matrix of the input voltages to the respective converters. $H$ is the Hamiltonian, which, in this case, is a quadratic function of $\boldsymbol{x}$, given as $H=\frac{1}{2} \boldsymbol{x}^{T} \mathbf{A} \boldsymbol{x}$, where $\mathbf{A}=\mathrm{I}_{\mathrm{n}}$.

$$
\begin{aligned}
& \mathbf{D}=\left[\begin{array}{cccc}
\mathbf{D}_{0} & 0 & \ldots & 0 \\
0 & \mathbf{D}_{1} & \cdots & 0 \\
\vdots & \vdots & \ddots & \vdots \\
0 & 0 & \ldots & \mathbf{D}_{\mathrm{m}}
\end{array}\right] \quad \mathbf{D}_{\mathrm{n}}\left[\begin{array}{ccc}
L_{\mathrm{n}} & 0 & 0 \\
0 & C_{\mathrm{n}} & 0 \\
0 & 0 & L_{T \mathrm{n}}
\end{array}\right] \\
& \boldsymbol{x}=\left[\begin{array}{c}
\boldsymbol{x}_{0} \\
\vdots \\
\boldsymbol{x}_{\mathrm{m}}
\end{array}\right] \quad \boldsymbol{x}_{\mathrm{n}}=\left[\begin{array}{c}
i_{L \mathrm{n}} \\
v_{C \mathrm{n}} \\
i_{T \mathrm{n}}
\end{array}\right] \\
& \mathbf{J}=\left[\begin{array}{cccccc}
\mathbf{A}_{0} & -\mathbf{B}^{T} & 0 & \ldots & 0 & \mathbf{B} \\
\mathbf{B} & \mathbf{A}_{1} & -\mathbf{B}^{T} & 0 & \ldots & 0 \\
0 & \mathbf{B} & \ddots & \ddots & \ddots & \vdots \\
\vdots & 0 & \ddots & \ddots & \ddots & 0 \\
0 & \vdots & \ddots & \ddots & \ddots & -\mathbf{B}^{T} \\
-\mathbf{B}^{T} & 0 & \cdots & 0 & \mathbf{B} & \mathbf{A}_{\mathrm{m}}
\end{array}\right] \\
& \text { Here, } \mathbf{A}_{\mathrm{n}}=\left[\begin{array}{ccc}
0 & -\left(1-u_{\mathrm{n}}\right) & 0 \\
\left(1-u_{\mathrm{n}}\right) & 0 & -1 \\
0 & 1 & 0
\end{array}\right], \mathbf{B}=\left[\begin{array}{lll}
0 & 0 & 0 \\
0 & 0 & 1 \\
0 & 0 & 0
\end{array}\right] \\
& \mathbf{R}=\left[\begin{array}{cccc}
\mathbf{R}_{0} & 0 & \ldots & 0 \\
0 & \mathbf{R}_{1} & \ldots & 0 \\
\vdots & \vdots & \ddots & \vdots \\
0 & 0 & \ldots & \mathbf{R}_{\mathrm{m}}
\end{array}\right], \mathbf{R}_{\mathrm{n}}=\left[\begin{array}{ccc}
0 & 0 & 0 \\
0 & \frac{1}{R_{2 T \mathrm{n}}} & 0 \\
0 & 0 & R_{1 T \mathrm{n}}
\end{array}\right] \\
& \mathbf{E}=\left[\begin{array}{c}
\mathbf{E}_{0} \\
\vdots \\
\mathbf{E}_{\mathrm{m}}
\end{array}\right], \mathbf{E}_{\mathrm{n}}=\left[\begin{array}{c}
E_{\mathrm{n}} \\
0 \\
0
\end{array}\right]
\end{aligned}
$$

Looking at the adjacency matrix of the interconnection $(\mathbf{J})$, the ring coupled structure is clearly verified. It is seen that $\mathbf{D}$ is a diagonal matrix and $\mathbf{R}$ is a symmetric matrix for $\mathrm{m}$ converters. The matrix $\mathbf{J}$ is the interconnection matrix and shows the coupling between neighbouring converters through dissipation. From Eq. (7), it can be verified that $\mathbf{J}$ is a skew-symmetric matrix keeping the PCHM structure [15]. 


\section{Application of passivity-based control}

The control objective for the ring coupled converter system is to regulate the output voltage of the ring towards a desired equilibrium value. First, we analyse the steady state behaviour of the system and then stabilize the system with a feedback control.

\subsection{Steady state equations of the system}

Till now the switched model for boost converters, given by $[13,16]$, has been considered. The control objective is to regulate the average output voltage to a constant reference (e.g DC/DC converters) or a periodic value with frequency much smaller than the switching frequency (inverters). Thus, it is desirable to consider the average value of the voltages and currents rather than the instantaneous values, given that the ripple and harmonics are sufficiently small.

The averaging of the circuit implies extracting the DC components of the circuit variables as well as the switching function which governs the switch position. In order to obtain the averaged model of the switching function, we replace the switch position $u_{\mathrm{n}}$ in the instantaneous circuit with a modulating signal $\mu_{\mathrm{n}}$ through averaging. A pulse width modulation (PWM) policy is implemented for switch regulation for the converters. PWM is a method for generating a digital pulse signal to drive the transistor switches in the boost converter circuit from a continuous control input or a modulating signal. If the modulating signal varies slowly in comparison to the switching frequency, it can be shown that the switching function is equal to the modulating signal [13]. As all the other components in the circuit are linear time invariant (LTI), they can be just replaced by their average values without any change. The only modification necessary is the replacement of the switching function with the modulating signal for the PWM, which will be referred to as the duty ratio. Hereafter, in this paper, the state variables $i_{L \mathrm{n}}, i_{T \mathrm{n}}, v_{C \mathrm{n}}$ will be considered as the average values of the instantaneous variables, and $\mu_{\mathrm{n}}$ as the duty ratio.

To gain insight into the steady state behaviour of the system, it is desirable to establish the relationship between the equilibrium values of the average output voltage, the average input current, and the average dissipation current. Towards this end, a constant duty ratio $\mu_{\mathrm{n}}=U_{\mathrm{n}}$ is implemented. Then, it is clear from Eqs. (1)-(3) that the equilibrium values of the state variables are given by Eqs. (11)-(13).

$$
\begin{aligned}
\bar{i}_{L \mathrm{n}}= & \frac{E_{\mathrm{n}}}{R_{2 T(\mathrm{n}-1)}\left(1-U_{\mathrm{n}}\right)^{2}}+\frac{1}{R_{1 T \mathrm{n}}}\left[\frac{E_{\mathrm{n}}}{\left(1-U_{\mathrm{n}}\right)^{2}}-\frac{E_{(\mathrm{n}+1)}}{\left(1-U_{\mathrm{n}+1}\right)\left(1-U_{\mathrm{n}}\right)}\right] \\
& -\frac{1}{R_{1 T(\mathrm{n}-1)}}\left[\frac{E_{(\mathrm{n}-1)}}{\left(1-U_{(\mathrm{n}-1)}\right)\left(1-U_{\mathrm{n}}\right)}-\frac{E_{\mathrm{n}}}{\left(1-U_{\mathrm{n}}\right)^{2}}\right] \\
\bar{v}_{C \mathrm{n}}= & \frac{E_{\mathrm{n}}}{\left(1-U_{\mathrm{n}}\right)} \\
\bar{i}_{T_{\mathrm{n}}}= & \frac{1}{R_{1 T \mathrm{n}}}\left[\frac{E_{\mathrm{n}}}{\left(1-U_{\mathrm{n}}\right)}-\frac{E_{(\mathrm{n}+1)}}{\left(1-U_{(\mathrm{n}+1)}\right)}\right]
\end{aligned}
$$

Here, $\bar{i}_{L \mathrm{n}}$ denotes steady state inductor current, $\bar{v}_{C \mathrm{n}}$ the steady state output voltage, and $\bar{i}_{T \mathrm{n}}$ the steady state dissipation current for converter \#n.

The desired output voltage $v_{C}^{*}$ decides the duty ratio. In other words, the duty ratio is used to keep the output in the ring at $\bar{v}_{C \mathrm{n}}=v_{C}^{*}$ for all $\mathrm{m}$ converters. This is an open loop system. The next section gives the estimation of the open loop system to compare it to the results of the closed loop system with PBC.

\subsection{Consideration of zero dynamics}

In this section we consider the analysis of the averaged ring coupled converter system through to the zero dynamics at the equilibrium points. Zero dynamics are defined as the dynamics that characterizes 


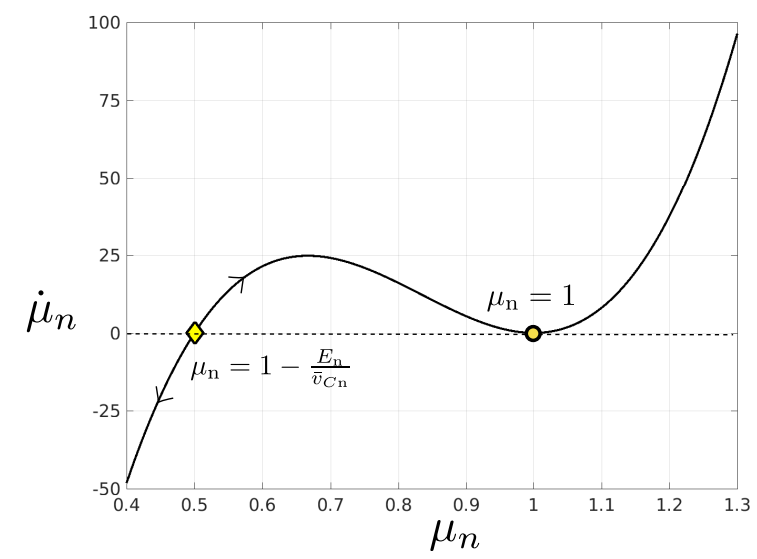

Fig. 3. Zero dynamics of ring coupled converter system corresponding average output capacitor voltage.

the internal behaviour of the system once the initial conditions and inputs are chosen, such that the output is identically zero [17]. Here, we consider the 'zero dynamics' associated with the equilibrium points given for the output as the capacitor voltage and the inductor current respectively.

Firstly, the output voltage of the capacitor is regarded as the output of the averaged PWM model of the ring coupled system. Then, rewriting the equations given by Eqs. (1)-(3) in terms of $v_{C n}$, the following relation is obtained.

$$
\begin{aligned}
C_{\mathrm{n}} \ddot{v}_{C \mathrm{n}}= & -\dot{\mu}_{\mathrm{n}} i_{L \mathrm{n}}+\left(1-\mu_{\mathrm{n}}\right) \dot{i}_{L \mathrm{n}}-\dot{i}_{T \mathrm{n}}+\dot{i}_{T \mathrm{n}}-\frac{\dot{v}_{C \mathrm{n}}}{R_{2 T(\mathrm{n}-1)}} \\
= & \frac{-\dot{\mu}_{\mathrm{n}}}{\left(1-\mu_{\mathrm{n}}\right)}\left[C_{\mathrm{n}} \dot{v}_{C \mathrm{n}}+i_{T \mathrm{n}}-i_{T(\mathrm{n}-1)}+\frac{v_{C \mathrm{n}}}{R_{1 T \mathrm{n}}}\right] \\
& +\frac{\left(1-\mu_{\mathrm{n}}\right)}{L_{\mathrm{n}}}\left[E_{\mathrm{n}}-\left(1-\mu_{\mathrm{n}}\right)\right]-\left(\dot{i}_{T \mathrm{n}}-\dot{i}_{T(\mathrm{n}-1)}\right)-\frac{\dot{v}_{C \mathrm{n}}}{R_{2 T(\mathrm{n}-1)}}
\end{aligned}
$$

The objective for the zero dynamics is to choose the control variable $\mu_{\mathrm{n}}$ so as to keep the output voltage as well as the dissipation current constrained as: $v_{C \mathrm{n}}=\bar{v}_{C \mathrm{n}}, i_{T \mathrm{n}}=\bar{i}_{T \mathrm{n}}$. Then, it can be conferred that $\ddot{v}_{C}=0$ and $\dot{v}_{C \mathrm{n}}=0$ as well as $\dot{i}_{T \mathrm{n}}=0$ and $\dot{i}_{T(\mathrm{n}-1)}=0$. Thus, the output is fixed but the control is not, and hence $\dot{\mu}_{\mathrm{n}} \neq 0$. Then, the following relationship is held.

$$
\dot{\mu}_{\mathrm{n}}=\frac{\left(1-\mu_{\mathrm{n}}\right)^{2}}{L_{\mathrm{n}}\left[\left(\bar{i}_{T \mathrm{n}}-\bar{i}_{T(\mathrm{n}-1)}\right)+\bar{v}_{C \mathrm{n}} / R_{2 T(\mathrm{n}-1)}\right]}\left[E_{\mathrm{n}}-\left(1-\mu_{\mathrm{n}}\right) \bar{v}_{C \mathrm{n}}\right]
$$

The equilibrium points of Eq. (16) are given at $\mu_{\mathrm{n}}=1-E_{\mathrm{n}} / \bar{v}_{C \mathrm{n}}$ and $\mu_{\mathrm{n}}=1$. Among them, the first has physical significance. If $\bar{v}_{C \mathrm{n}}>E_{\mathrm{n}}$, it is confirmed that the output will be always higher than the input of the converter. However, the phase diagram describes this equilibrium point as unstable, making the system non-minimum phase with respect to the output voltage. The phase diagram is drawn by Fig. 3 in accordance with the parameters given in Table I. Such analysis is shown possible for single boost converter in [4] and [16].

Next, the analysis of the system corresponding to the zero dynamics with the inductor current as the output of the averaged PWM model is carried out. Then, rewriting the equations given by Eqs. (1)-(3) in terms of $i_{L \mathrm{n}}$ the following equation is obtained.

$$
L_{\mathrm{n}} \ddot{i}_{L \mathrm{n}}=\dot{\mu}_{\mathrm{n}}\left[\frac{E_{\mathrm{n}}-L_{\mathrm{n}} \dot{i}_{L \mathrm{n}}}{\left(1-\mu_{\mathrm{n}}\right)}\right]-\frac{\left(1-\mu_{\mathrm{n}}\right)}{L_{\mathrm{n}} C_{\mathrm{n}}}\left[\left(1-\mu_{\mathrm{n}}\right) i_{L \mathrm{n}}-i_{T \mathrm{n}}+i_{T(\mathrm{n}-1)}-\frac{E_{\mathrm{n}}-L_{\mathrm{n}} \dot{i}_{L \mathrm{n}}}{\left(1-\mu_{\mathrm{n}}\right) R_{2 T(\mathrm{n}-1)}}\right]
$$

Same as above, the control variable $\mu_{\mathrm{n}}$ can be chosen so as to keep the output at a constant value $i_{L \mathrm{n}}=\bar{i}_{L \mathrm{n}}$ which implies that $i_{T \mathrm{n}}=\bar{i}_{T \mathrm{n}}$. Then, it follows that $\ddot{i}_{L \mathrm{n}}=0$ and $\dot{i}_{L \mathrm{n}}=0$. The remaining dynamics related to the control variable, i.e. the duty ratio $\mu_{\mathrm{n}}$ are described by Eq. (18). 


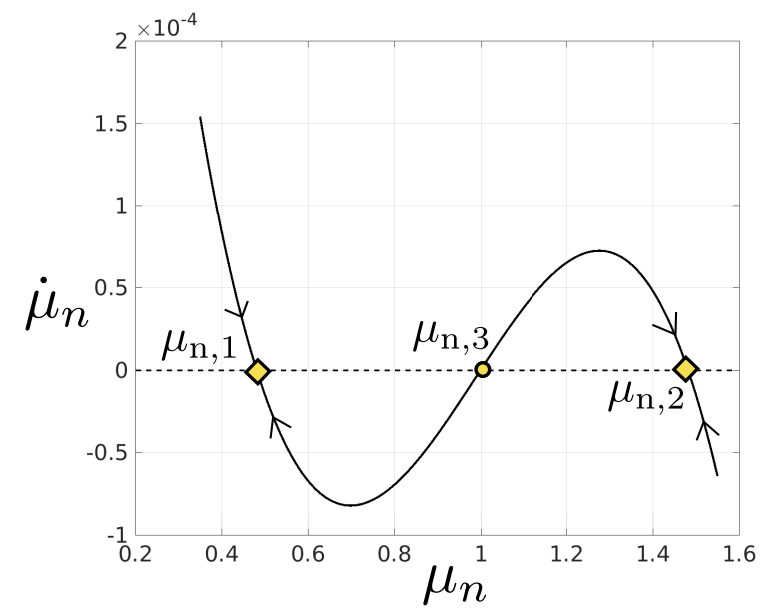

Fig. 4. Zero dynamics of ring coupled converter system corresponding to average of output inductor current.

$$
\dot{\mu}_{\mathrm{n}}=\frac{\left(1-\mu_{\mathrm{n}}\right)^{2}}{L_{\mathrm{n}}\left[\left(\bar{i}_{T \mathrm{n}}-\bar{i}_{T(\mathrm{n}-1)}\right)+\bar{v}_{C \mathrm{n}} / R_{2 T(\mathrm{n}-1)}\right]}\left[E_{\mathrm{n}}-\left(1-\mu_{\mathrm{n}}\right) \bar{v}_{C \mathrm{n}}\right]
$$

The three equilibrium points, $\left\{\mu_{n, i}\right\}_{i=1,2,3}$, corresponding to Eq. (18), are obtained as Eq. (19).

$$
\begin{aligned}
& \mu_{\mathrm{n}, 1}=1-\frac{\left(\bar{i}_{T \mathrm{n}}-\bar{i}_{T(\mathrm{n}-1)}\right)}{2 \bar{i}_{L \mathrm{n}}}-\sqrt{\frac{E_{\mathrm{n}}}{R_{2 T(\mathrm{n}-1)} \bar{i}_{L \mathrm{n}}}-\left[\frac{\left(\bar{i}_{T \mathrm{n}}-\bar{i}_{T(\mathrm{n}-1)}\right)^{2}}{4\left(\bar{i}_{L \mathrm{n}}\right)^{2}}\right]} \\
& \mu_{\mathrm{n}, 2}=1-\frac{\left(\bar{i}_{T \mathrm{n}}-\bar{i}_{T(\mathrm{n}-1)}\right)}{2 \bar{i}_{L \mathrm{n}}}+\sqrt{\frac{E_{\mathrm{n}}}{R_{2 T(\mathrm{n}-1)} \bar{i}_{L \mathrm{n}}}-\left[\frac{\left(\bar{i}_{T \mathrm{n}}-\bar{i}_{T(\mathrm{n}-1))^{2}}\right.}{4\left(\bar{i}_{L \mathrm{n}}\right)^{2}}\right]} \\
& \mu_{\mathrm{n}, 3}=1
\end{aligned}
$$

As $\left(\bar{i}_{T \mathrm{n}}-\bar{i}_{T(\mathrm{n}-1)}\right)$ is sufficiently small, we can safely neglect it in the squared terms. It can also be established that $\left(\bar{i}_{T \mathrm{n}}-\bar{i}_{T(\mathrm{n}-1)}\right) / 2 \bar{i}_{L \mathrm{n}}<<\sqrt{E_{\mathrm{n}} / R_{2 T \mathrm{n}} \bar{i}_{L \mathrm{n}}}$. Thus, only one equilibrium point satisfies the condition of $0 \leq \mu_{\mathrm{n}}<1$ and is of physical significance. All the equilibrium points are given by Fig. 4, in accordance with the parameters specified in Table I. It is seen that this equilibrium point is stable, as long as $R_{2 T \mathrm{n}} \bar{i}_{L \mathrm{n}}>E_{\mathrm{n}}$. This again emphasizes the properties of the boost converter, making the system controlled with inductor current, a minimum phase system. The inductor current will be used to stabilize the system rather than the output capacitor voltage.

\subsection{Energy shaping in PBC}

We investigate whether it is possible for PBC to be applied to a system of multiple converters coupled with dissipation. The stored energy of a circuit is the sum of the energy in the passive elements, that is the inductors and capacitors. The energy of the multiple converter system is given by Eq. (20).

$$
H=\frac{1}{2} \boldsymbol{x}^{T} \mathbf{D} \boldsymbol{x}
$$

$\boldsymbol{x}$ and $\mathbf{D}$ are as given in Eqs. (5) and (6), respectively. $H$, which is the total energy, consisting of the kinetic energy through the inductor and the potential energy stored in the capacitor.

The desired energy function is designed based on the total energy of the system. The formulation is taken to be the quadratic function of errors. It is given by Eq. (21).

$$
H_{d}=\frac{1}{2} \boldsymbol{e}^{T} \mathbf{D} \boldsymbol{e}, \quad \boldsymbol{e}=\boldsymbol{x}-\boldsymbol{x}_{d}
$$

Here, $\boldsymbol{x}_{\boldsymbol{d}}=\left[\begin{array}{lll}\boldsymbol{x}_{1 d} & \boldsymbol{x}_{2 d} \ldots \boldsymbol{x}_{\mathrm{m} d}\end{array}\right]^{T}$ is the desired trajectory of the state. Here, $\boldsymbol{x}_{\mathrm{n} d}=\left[\begin{array}{l}\bar{i}_{L \mathrm{n}} v_{C \mathrm{n} d} i_{T \mathrm{n} d}\end{array}\right]^{T}$, where $\bar{i}_{L \mathrm{n}}$ is a constant, $v_{C \mathrm{n} d}(t)$ and $i_{T \mathrm{n} d}(t)$ are the desired output voltage and line currents. In order 
to prove that the function given in Eq. (21) is a candidate of Lyapunov function, let us check the derivative of the energy function as in Eq. (22).

$$
\begin{aligned}
\dot{H}_{d} & =\left(\boldsymbol{x}-\boldsymbol{x}_{d}(t)\right)^{T} \mathbf{D}\left(\dot{\boldsymbol{x}}-\dot{\boldsymbol{x}}_{d}(t)\right) \\
& =\left(\boldsymbol{x}-\boldsymbol{x}_{d}(t)\right)^{T}\left([\mathbf{J}-\mathbf{R}] \boldsymbol{x}+\mathbf{E}-\mathbf{D} \dot{\boldsymbol{x}}_{d}(t)\right)
\end{aligned}
$$

By setting the term $\mathbf{D} \dot{\boldsymbol{x}}_{d}(t)$ as given in Eq. (23), the control rule with PBC can be formulated for boost converters.

$$
\mathbf{D} \dot{\boldsymbol{x}}_{d}(t)=(\mathbf{J}-\mathbf{R}) \boldsymbol{x}_{d}(t)+\mathbf{E}+\mathbf{R}_{I}\left(\boldsymbol{x}-\boldsymbol{x}_{d}(t)\right)
$$

Where, $\mathbf{R}_{I}$ is a symmetric and positively defined matrix, which acts as the damping injection, making the system asymptotically stable if $\mathbf{R}+\mathbf{R}_{I}>0$. The damping is added via the controller, thus helping to determine the duty ratio after feedback. The damping injection complements the dissipation of the original system [11]. By considering that $\boldsymbol{e}^{T} \mathbf{J}(\mu) \boldsymbol{e}=0$ for all values of $\mu$, we obtain the following condition on the derivative of our chosen Lyapunov function $H_{d}(\boldsymbol{e})$.

$$
\dot{H}_{d}(e)=\boldsymbol{e}^{T}\left(\mathbf{J}(\mu) \boldsymbol{e}-\mathbf{R} \boldsymbol{e}-\mathbf{R}_{I} \boldsymbol{e}\right)=-\boldsymbol{e}^{T}\left(\mathbf{R}+\mathbf{R}_{I}\right) \boldsymbol{e}<0
$$

Under the control rule, the error $\boldsymbol{e}$ converges to the origin asymptotically. Then, by satisfying the condition $\left(\mathbf{R}+\mathbf{R}_{I}\right)>0$, the system becomes exponentially and asymptotically stable at the equilibrium point [18]. Then, the derivative of $H_{d}(e)$ is given in Eq. (25).

$$
\dot{H}_{d}(\boldsymbol{e})=-\boldsymbol{e}^{T}\left(\mathbf{R}+\mathbf{R}_{I}\right) \boldsymbol{e} \leq-k H_{d}(\boldsymbol{e})
$$

Hence, it is proved that Lyapunov's Theorem is satisfied. That is, the equilibrium state $\boldsymbol{x}_{d}$ is asymptotically stable with the control rule given in Eq. (23), and exponentially asymptotically stable with the damping injection. $\mathbf{R}_{I}$ is given in Eq. (26). The damping injection is a diagonal matrix of the damping matrices. The damping matrix for the $\# \mathrm{n}$ converter in the system is represented by $\mathbf{R}_{I \mathrm{n}}$.

$$
\mathbf{R}_{I}=\left[\begin{array}{cccc}
\mathbf{R}_{I 0} & 0 & \ldots & 0 \\
0 & \mathbf{R}_{I 1} & \ldots & 0 \\
\vdots & \vdots & \ddots & \vdots \\
0 & 0 & \ldots & \mathbf{R}_{I \mathrm{~m}}
\end{array}\right] \text {, where } \mathbf{R}_{I \mathrm{n}}=\left[\begin{array}{ccc}
R_{\mathrm{n}} & 0 & 0 \\
0 & 0 & 0 \\
0 & 0 & 0
\end{array}\right]
$$

Then, the control equation is obtained for the given system in Eq. (27). The boost converter is controlled with inductor current keeping the minimum phase nature as given by the zero dynamics. The constant desired inductor current $\left(\bar{i}_{L n}\right)$ is obtained by solving the steady state Eq. (11).

$$
\begin{aligned}
\mu_{\mathrm{n}} & =\frac{1}{v_{C \mathrm{n} d}}\left[E_{\mathrm{n}}+R_{\mathrm{n}}\left(i_{L \mathrm{n}}-\bar{i}_{L \mathrm{n}}\right)\right]+1 \\
C_{\mathrm{n}} \dot{v}_{C \mathrm{n} d} & =\left(1-\mu_{\mathrm{n}}\right) \bar{i}_{L \mathrm{n}}-i_{T_{\mathrm{n} d}}+i_{T(\mathrm{n}-1) d}-\left[\frac{v_{C \mathrm{n} d}}{R_{2 T(\mathrm{n}-1)}}\right] \\
L_{T_{\mathrm{n}}} \dot{i}_{T \mathrm{n} d} & =v_{C \mathrm{n} d}-v_{C((\mathrm{n}+1)) d}-R_{1 T \mathrm{n}} i_{T \mathrm{n} d}
\end{aligned}
$$

The value of the duty ratio $\mu_{\mathrm{n}}$ is evaluated at every instant $t$ depending on the input, the parameters, and the desired output of the system. That is, $\mu_{\mathrm{n}}$ depends on time and the state. Hereafter, when $\mathrm{PBC}$ is applied to the system, $\mu_{\mathrm{n}}(t)$ is considered as a function of time.

\section{Numerical simulations}

The desired voltage is set as $\bar{v}_{C n}$. Via Eqs. (11)-(13), the associated desired current $\bar{i}_{L \mathrm{n}}$ is calculated. From this, the corresponding desired trajectory $\boldsymbol{x}_{d}(t)$ is calculated by solving Eqs. (27)-(29). When the operator wants to set a desired voltage, only the current via the duty ratio is directly controllable. Hence it is necessary to convert the problem of obtaining the desired voltage, into a control problem of 
Table I. Parameters for Balanced System.

\begin{tabular}{lll}
\hline Parameter & Value & Unit \\
\hline$E_{\mathrm{n}}$ & 15 & $\mathrm{~V}$ \\
\hline$L_{\mathrm{n}}$ & 46 & $\mathrm{mH}$ \\
\hline$C_{\mathrm{n}}$ & 100 & $\mu \mathrm{F}$ \\
\hline$L_{T \mathrm{n}}$ & 15 & $\mathrm{mH}$ \\
\hline$R_{1 T \mathrm{n}}$ & 100 & $\Omega$ \\
\hline$R_{2 T \mathrm{n}}$ & 170 & $\Omega$ \\
\hline $\bar{v}_{C \mathrm{n}}$ & 40 & $\mathrm{~V}$
\end{tabular}
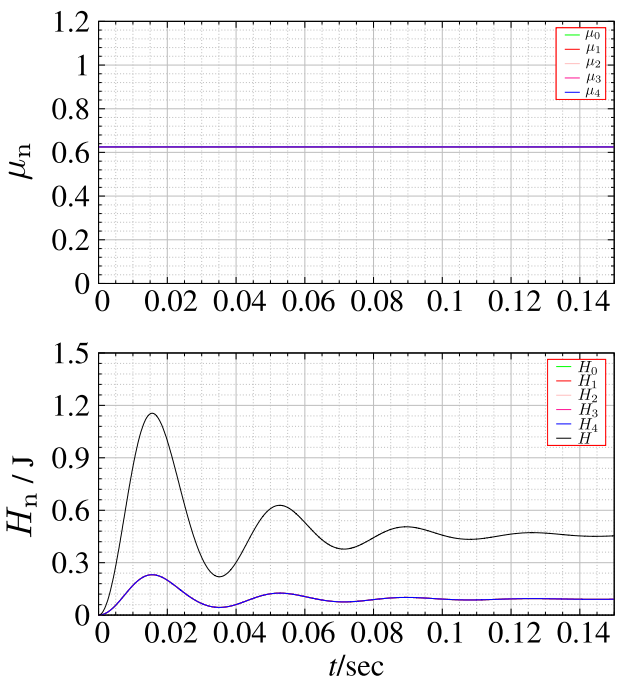

Table II. Different Input Voltages.

\begin{tabular}{lll}
\hline Parameter & Value & Unit \\
\hline$E_{0-4}$ & $15,13,12,13,15$ & $\mathrm{~V}$ \\
\hline$L_{\mathrm{n}}$ & 46 & $\mathrm{mH}$ \\
\hline$C_{\mathrm{n}}$ & 100 & $\mu \mathrm{F}$ \\
\hline$L_{T \mathrm{n}}$ & 15 & $\mathrm{mH}$ \\
\hline$R_{1 T \mathrm{n}}$ & 100 & $\Omega$ \\
\hline$R_{2 T \mathrm{n}}$ & 170 & $\Omega$ \\
\hline $\bar{v}_{C \mathrm{n}}$ & 40 & $\mathrm{~V}$
\end{tabular}
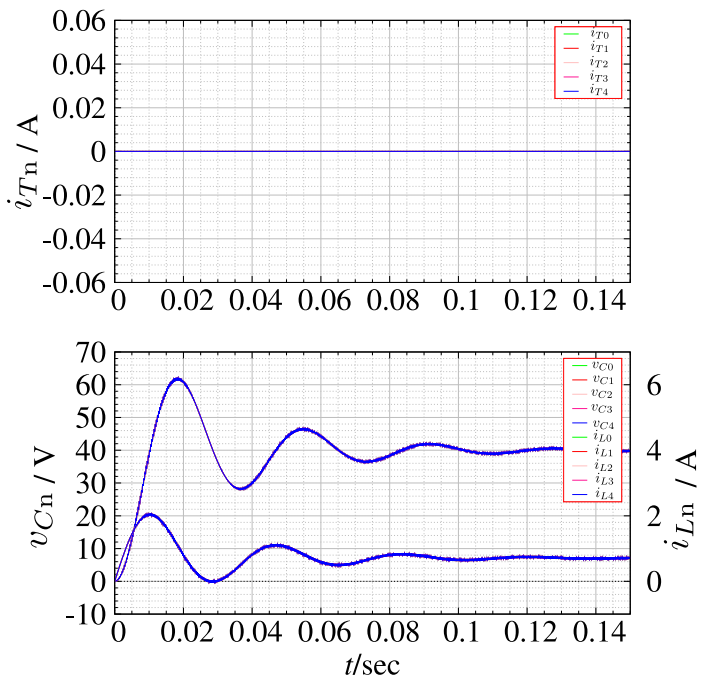

Fig. 5. Balanced system behaviour of original system with respect to time. Parameters of all converters are set at equal values. The desired output voltage $\bar{v}_{C}=40 \mathrm{~V}$ for all 5 converters. The output of all converters coincides through the transient and settles at $40 \mathrm{~V}$.

the current. Consequently, in the transient dynamics, the desired voltage, $v_{C n d}$, will remain fluctuating around $\bar{v}_{C \mathrm{n}}$.

The simulation results obtained for five converters coupled in a ring form are given in this section. The numerical simulations were carried out on ode45 solver Simulink (Version 8.7 R2016a).

\subsection{Simulation results for a balanced system}

Parameters in the ring coupled converter system are set at the same values respectively. This is a balanced case. Additionally, the dissipation between the converters $\left(L_{T \mathrm{n}}, R_{1 T \mathrm{n}}\right)$ as well as the inputs $E_{\mathrm{n}}$ are also set at same values for all the converters. This naturally creates an energy balance in the ring. To begin with, numerical simulations are performed for a balanced system without applying any feedback control. Then, the duty cycle is set at a constant value $\left(U_{\mathrm{n}}\right)$. This value is calculated by Eq. (12) for a particular $\bar{v}_{C}$. Thus, any disturbance in the system will not be acted on but will affect the system output adversely. The parameters for simulation are given in Table I. Figure 5 shows the inductor current, dissipation current, output voltage, and duty cycle with respect to time. In the transient, the output voltage and the inductor current shows the transient oscillations. After the transient, the system reaches an equilibrium. The equilibrium value of the output voltage becomes equal to $\bar{v}_{C}$. As all the boost converters have same inputs and same parameter values, no current flows through the dissipation.

Next we look at simulations results when PBC is applied. PBC results in an output dynamic feedback controller which induces a shaped closed loop energy and enhances the closed loop damping of the system. The damping has the condition $\mathbf{R}+\mathbf{R}_{I}>0$. Then, it was set and kept at $\mathbf{R}_{I 1}=15$ for all the simulations. The results, for a balanced case, show faster response and damped oscillations. Feedback is provided by solving Eq. (27) to obtain the appropriate duty cycle value to maintain the 

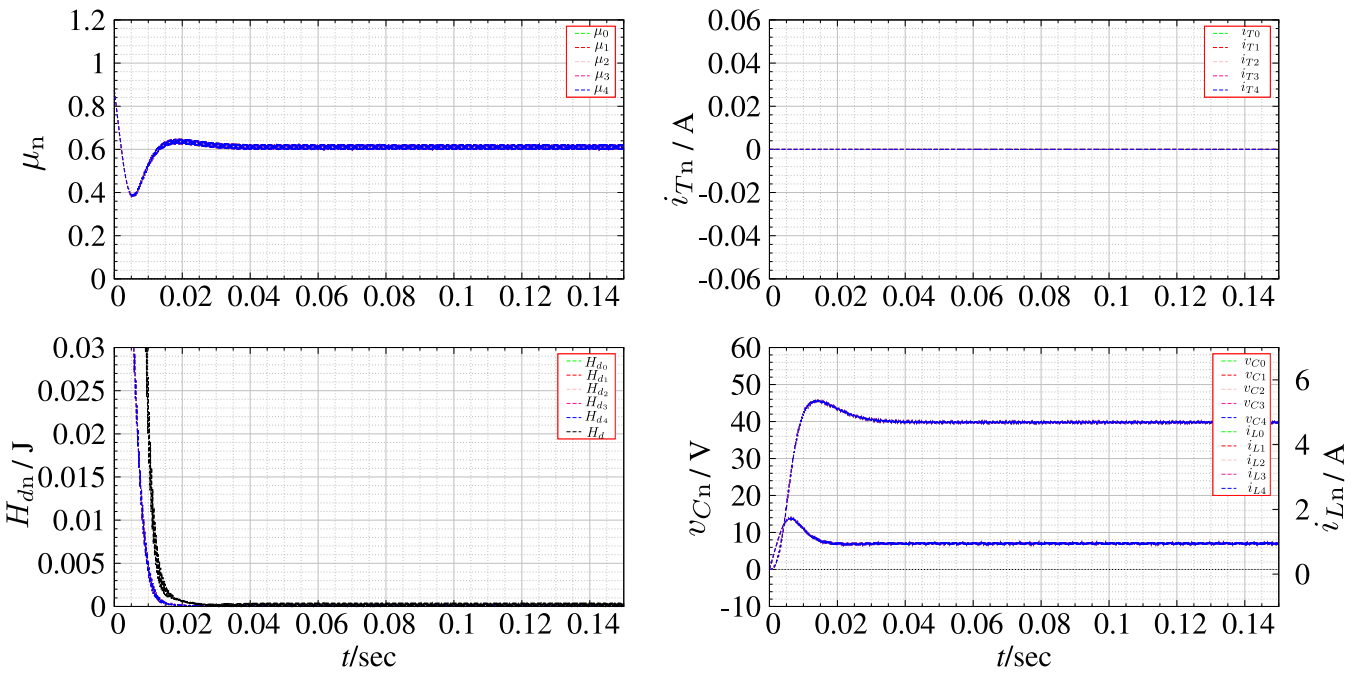

Fig. 6. Balanced system behaviour with application of $\mathrm{PBC}$ to all converters. The energy function $H_{d}$ goes to zero as system settles to the desired equilibrium. As the system is balanced, all the converters move synchronously. The transient peak is damped and the convergence time is improved.

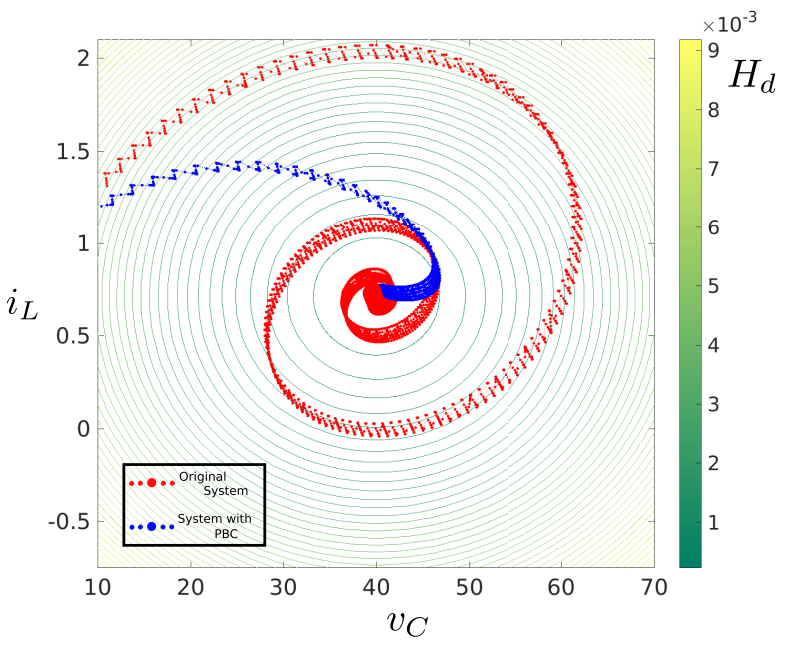

Fig. 7. Asymptotic behaviour of the original system and system with PBC.

desired equilibrium voltage. Here, the inductor current is the variable that is measured to provide feedback. The numerical simulation results are shown in Fig. 6.

The simulation parameters are set as given in Table I. Comparing Fig. 6 with Fig. 5, it is seen that the transient is sufficiently dampened. The time to stabilize the system to the equilibrium is also significantly faster. As all the parameters are the same for all boost converter systems as well as the dissipation, the trajectories on the output voltage and inductor current plane coincide for all five converters. The asymptotic behaviour is clearly observed with a phase plot on the energy sets of $H_{d}$. This is shown in Fig. 7.

\subsection{Simulation results for an unbalanced system}

Imbalance occurs in the ring coupled system when converters in the ring and/or the dissipation between two neighbouring converters have different parameter values. In the following simulations we consider imbalance in two different ways: imbalance created with varying input voltage and load resistance values. First, simulations were carried out to see the behaviour of the original unbalanced system without application of PBC. The values of the input voltages are varied as given in Table II.

Figure 8 shows that different input voltages give rise to different current values for the same desired voltage. In this case, all converters have constant but different duty cycles as calculated from Eq. (12). 

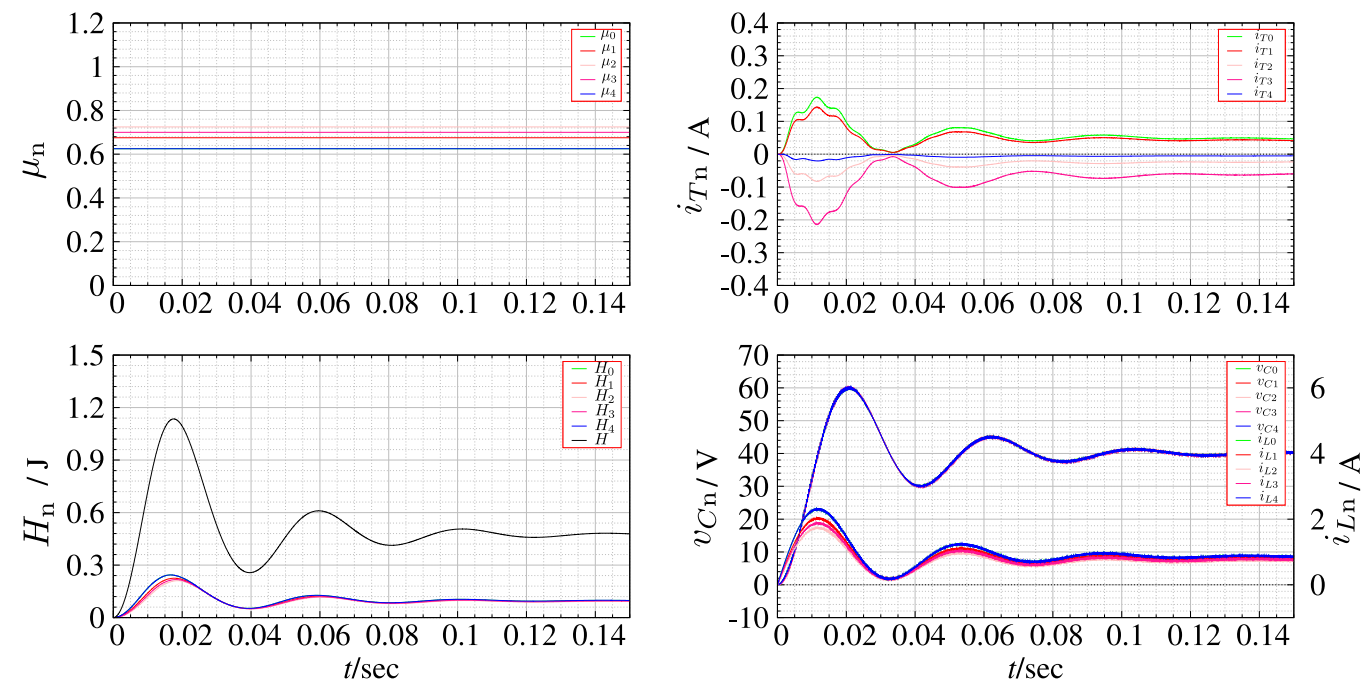

Fig. 8. Original unbalanced system with different input voltages to the boost converter. Imbalance is created with input values given in Table II.
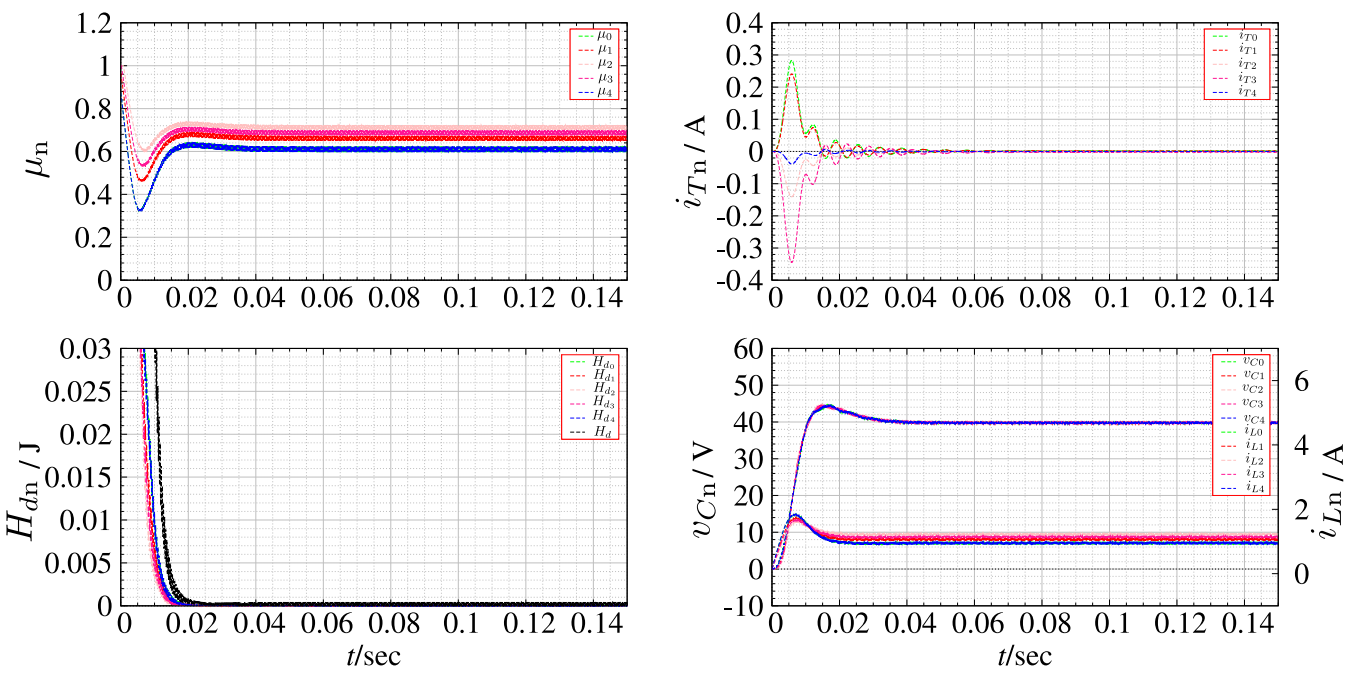

Fig. 9. Unbalanced system controlled with $\mathrm{PBC}$ having different input voltages for the boost converters. Imbalance is created with input values given in Table II.

Due to the different inputs, it is found that current appears through the dissipation between the coupled converters. The dissipation current is reduced after the transient when the converters converge to the desired voltage.

Figure 9 shows simulation results when PBC is applied. The direction of flow of the dissipation current depends on the imbalance created by the different inputs. The duty ratio changes according to the energy function $\left(H_{d}\right)$ and becomes constant as soon as the energy function attains a zero value. The energy function for each of the converters is different for the unbalanced case. It is seen in the results that each energy function becomes zero as the control is applied. This implies that PBC is successfully applied to each converter system as well. Thus, it can be confirmed that interconnection of passive systems is a passive system [3]. Even though energy is exchanged between converter systems during transient period, passivity is retained for each converter, and all converters stabilize at the desired equilibrium.

Next, the load resistances are set differently for each of the converters as given in Table III. The simulation results for the original system are shown in Fig. 10. The different load resistances cause the voltages to settle at slightly different values, this difference is unrecognizable but can be inferred from the continuous flow of dissipation current between the converter systems. This indicates the flow of energy between the converters. The flow of energy is in the direction of the load that consumes the 
Table III. Different Load Resistances.

\begin{tabular}{lll}
\hline Parameter & Value & Unit \\
\hline$E_{0-4}$ & 15 & $\mathrm{~V}$ \\
\hline$L_{\mathrm{n}}$ & 46 & $\mathrm{mH}$ \\
\hline$C_{\mathrm{n}}$ & 100 & $\mu \mathrm{F}$ \\
\hline$L_{T \mathrm{n}}$ & 15 & $\mathrm{mH}$ \\
\hline$R_{1 T \mathrm{n}}$ & 100 & $\Omega$ \\
\hline$R_{2 T 0-4}$ & $130,170,140,170,130$ & $\Omega$ \\
\hline $\bar{v}_{C \mathrm{n}}$ & 40 & $\mathrm{~V}$
\end{tabular}
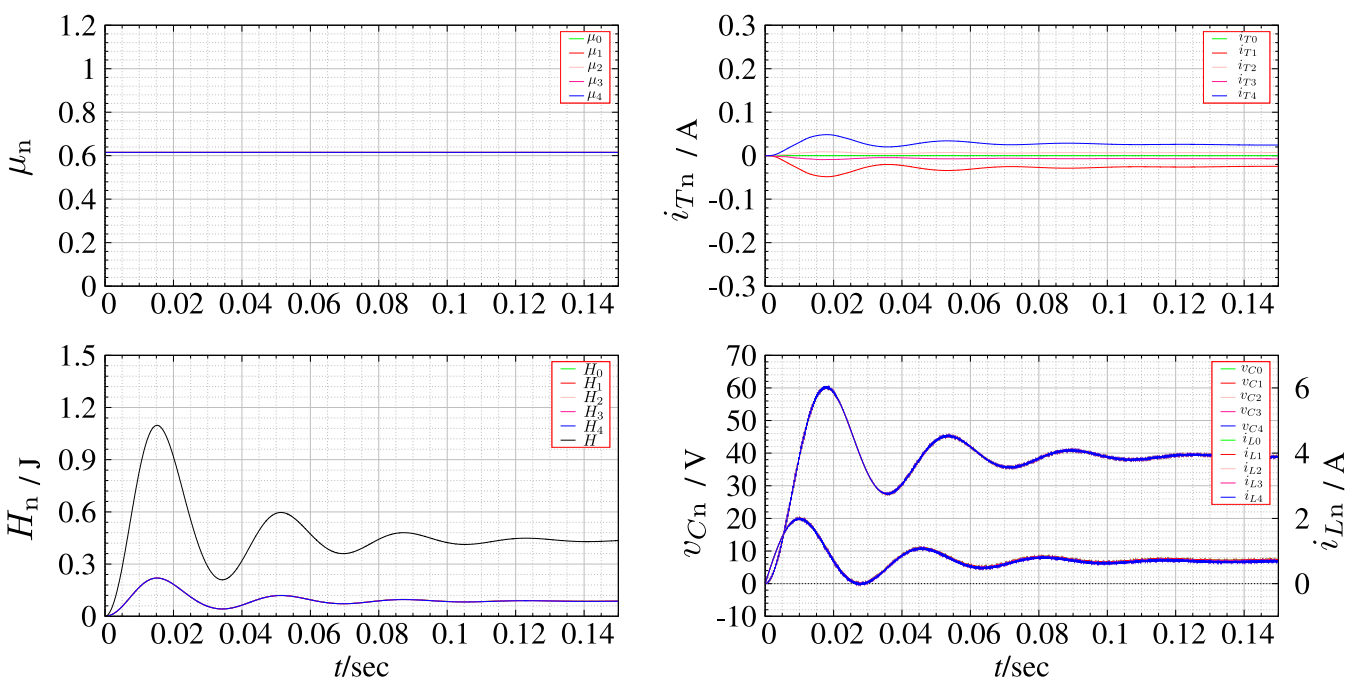

Fig. 10. Original system with imbalance created with different load resistance values as given in Table III.
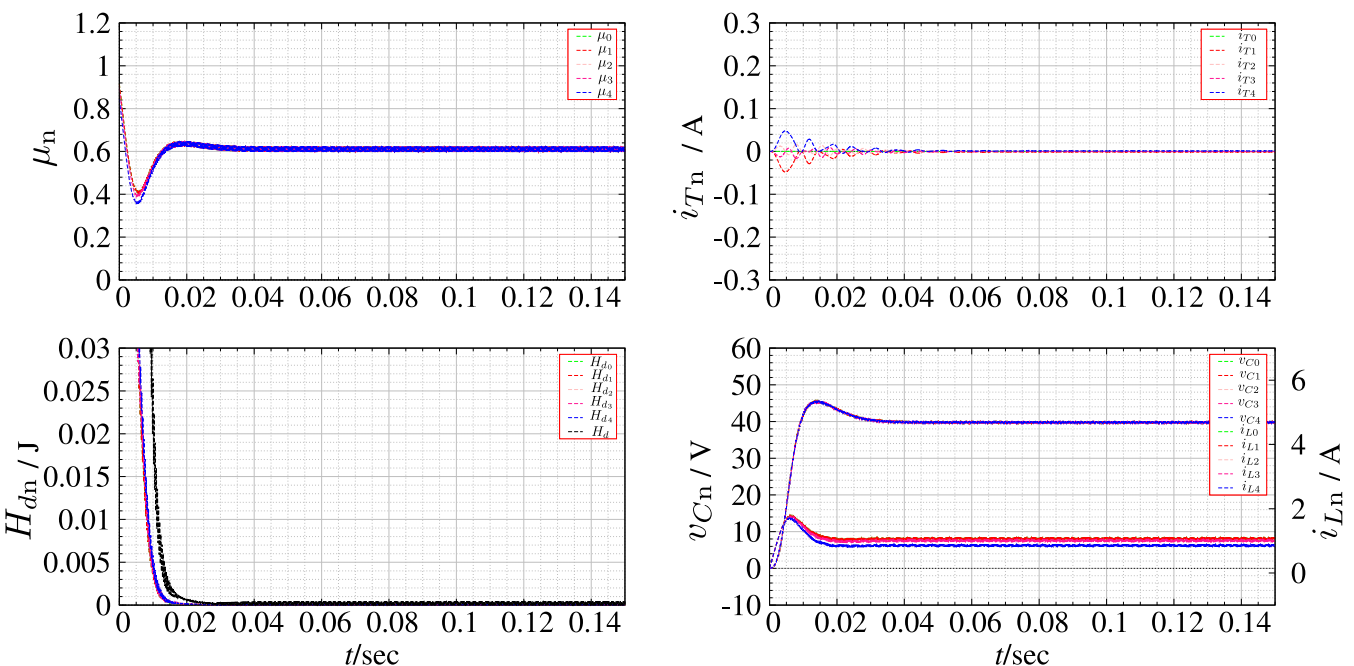

Fig. 11. Unbalanced system controlled with PBC having different load resistance values as given in Table III.

most current, i.e towards the smallest resistance.

Finally, Fig. 11 shows the results of PBC applied to the system when the load resistance values are different. As is expected, the results are better than the uncontrolled case. This implies that PBC can successfully regulate the coupled converter systems when the system has different loads. The loads in a realistic system would vary depending on their power demand. Therefore, the successful stabilization of the system with PBC with different loads serves as an useful tool for designing practical systems. 


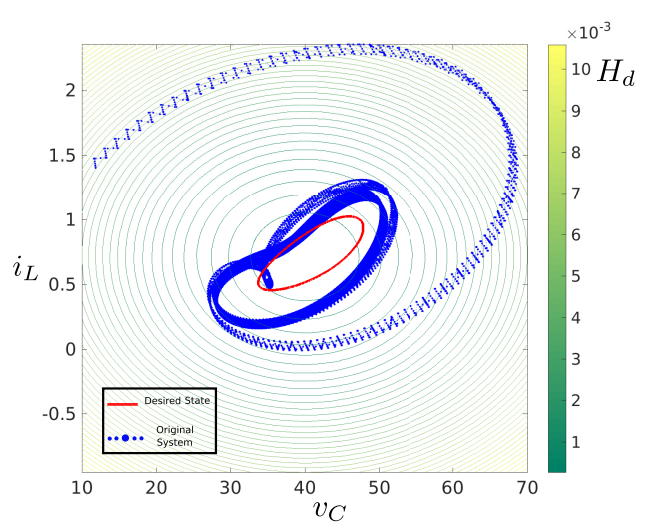

(a)

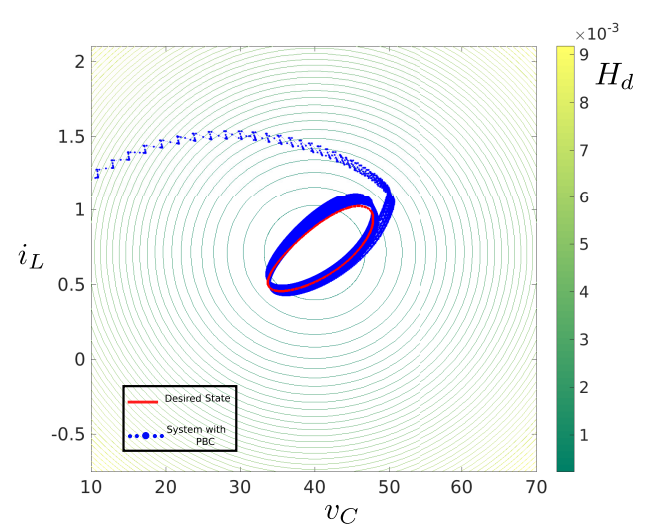

(b)

Fig. 12. Asymptotic behaviour of system with the desired equilibrium as a function of time: (a) Without application of control (b) With application of PBC.

\subsection{Stabilization to non stationary state}

So far the control has been constructed assuming that the desired state, $v_{C}^{*}=\bar{v}_{C}$ is a constant. We may further extend PBC to the case in which the desired state is not a constant but a function on time. A sinusoidal function with a DC bias is selected as the desired state. The desired state is set to be $v_{C}^{*}=v_{D C}+A \sin (2 \pi f t)$. Here, $v_{D C}$ is the DC bias voltage, $A$ and $f$ are the amplitude and frequency of the sinusoidal voltage. The frequency is much smaller than the switching frequency of the PWM. For simulations we set $v_{D C}=40 \mathrm{~V}, A=8 \mathrm{~V}$, and $f=60 \mathrm{~Hz}$. Firstly, the simulations were carried out for the original system, where the duty ratio $U_{\mathrm{n}}$ is calculated from Eq. (12). The results are shown in the form of an energy plot (Fig. 12(a)). The other parameters are set as given by Table I.

Next, simulations were carried out by applying PBC as given by Eqs. (27) and (29). The results are given in Fig. 12(b). Again, the parameters are set as given by Table I.

It was seen in Sec. 3.3, that the desired function is considered as a function of time. The subsequently obtained control equations for the feedback through the duty cycle also emphasize the fact that it is permissible to have the desired state as a function of time. It implies that PBC is a suitable method of control for such a case. The simulation results confirm the improvement at the application of PBC. The open loop system exhibits an unstable transient and a high transient peak voltage. The system with control settles down to the desired equilibrium relatively faster.

\section{Conclusion}

In this paper we proposed a method to stabilize a ring coupled converter system, consisting of DC/DC boost converters, to a desired state with the application of PBC. PBC, with energy shaping and damping injection, was discussed for the quadratic function of errors as the desired storage function. The desired storage function deviates around zero and finally approaches zero as the system attains equilibrium.

Numerical simulations show that PBC can stabilize the output voltage values at the desired state even for a ring coupled configuration of the DC/DC converters. Comparison to the dynamic behaviour of the original system suggests the successful application of PBC during transient operation. Numerical simulations were carried for different initial conditions. This included a balanced condition, where all the boost converters have same parameters, an unbalanced state with different parameters, including the dissipation, and the case in which the desired state is non stationary. PBC was applied for all the three cases. The results for the balanced system show all converters in the ring operating synchronously. There is no energy exchange in the form of dissipation current. Imbalance causes energy imbalance, but the application of $\mathrm{PBC}$ restores this imbalance and the entire system stabilizes at the desired state. Practically, the input voltages to the converters as well as the load resistances are not same for all converters in the ring. Even in such imbalanced conditions, the converters co- 
operate to maintain a stable voltage through the ring coupling. For non stationary desired states, the convergence of the output voltage as well as the inductor current to the desired sinusoidal state is vastly improved under PBC as compared to the original system, with a dampened transient peak.

\section{References}

[1] L.S.T. Ackermann and G. Andersson, "Distributed generation: a definition," Electric Power System Research, vol. 57, no. 3, pp. 195-204, 2001.

[2] Y. Takatsuji, Y. Susuki, and T. Hikihara, "Hybrid controller for safe microgrid operation," NOLTA, vol. 2, no. 3, pp. 347-362, 2011.

[3] P.L. Jie Bao, Process Control - The Passive Systems Approach, Springer-Verlag, 2007.

[4] R. Ortega, J.A.L. Perez, P.J. Nicklasson, and H. Sira-Ramirez, Passivity-based control of EulerLagrange systems: mechanical, electrical and electromechanical applications, Springer Science \& Business Media, 2013.

[5] V.M. Popov and R. Georgescu, "Hyperstability of control systems," 1973.

[6] J.C. Willems, "Dissipative dynamical systems part i: General theory," Archive for rational mechanics and analysis, vol. 45, no. 5, pp. 321-351, 1972.

[7] S.A. Morikazu Takegaki, "A new feedback method for dynamic control of manipulators," Journal of Dynamic Systems, Measurement, and Control, vol. 103, no. 2, 1980.

[8] R. Ortega and M.W. Spong, "Adaptive motion control of rigid robots: A tutorial," Automatica, vol. 25, no. 6, pp. 877-888, 1989.

[9] I.D. Landau and R. Horowitz, "Synthesis of adaptive controllers for robot manipulators using a passive feedback systems approach," in Robotics and Automation, 1988. Proceedings., 1988 IEEE International Conference on, pp. 1028-1033, IEEE, 1988.

[10] G. Escobar, A.J. Van Der Schaft, and R. Ortega, "A hamiltonian viewpoint in the modeling of switching power converters," Automatica, vol. 35, no. 3, pp. 445-452, 1999.

[11] H. Sira-Ramirez and R. Ortega, "Passivity-based controllers for the stabilization of dc-to-dc power converters," in Decision and Control, 1995., Proceedings of the 34th IEEE Conference on, vol. 4, pp. 3471-3476, IEEE, 1995.

[12] T. Hikihara and Y. Murakami, "Regulation of parallel converters with respect to stored energy and passivity characteristics," IEICE transactions on fundamentals of electronics, communications and computer sciences, vol. 94, no. 3, pp. 1010-1014, 2011.

[13] J.G. Kassakian, M.F. Schlecht, and G.C. Verghese, Principles of power electronics, vol. 1991, Addison-Wesley Reading, USA, 1991.

[14] B. Maschke and A. Van Der Schaft, "Port-controlled hamiltonian systems: modelling origins and systemtheoretic properties," IFAC Proceedings Volumes, vol. 25, no. 13, pp. 359-365, 1992.

[15] A. Van Der Schaft, "Port-hamiltonian systems: network modeling and control of nonlinear physical systems," in Advanced dynamics and control of structures and machines, pp. 127-167, Springer, 2004.

[16] H. Sira-Ramírez and R. Silva-Ortigoza, Control design techniques in power electronics devices, Springer Science \& Business Media, 2006.

[17] A. Isidori, "The zero dynamics of a nonlinear system: From the origin to the latest progresses of a long successful story," European Journal of Control, vol. 19, no. 5, pp. 369-378, 2013.

[18] H.K. Khalil, "Noninear systems," Prentice-Hall, New Jersey, vol. 2, no. 5, pp. 5-10, 1996. 